\title{
Why Bees Are So Vulnerable to Environmental Stressors
}

Simon Klein ${ }^{1,2,3}$, Amélie Cabirol ${ }^{1,2,3}$, Jean-Marc Devaud ${ }^{1}$, Andrew B. Barron ${ }^{2}$ and Mathieu Lihoreau ${ }^{1,}$

${ }^{1}$ Research Center on Animal Cognition, Center for Integrative Biology, National Center for Scientific Research (CNRS), University Paul Sabatier(UPS), Toulouse, France

${ }^{2}$ Department of Biological Sciences, Macquarie University, Sydney, NSW, Australia

${ }^{3}$ These authors contributed equally to this work.

*Correspondence: mathieu.lihoreau@univ-tlse3.fr

Bee populations are declining in the industrialized world, raising concerns for the sustainable pollination of crops. Pesticides, pollutants, parasites, diseases, and malnutrition have all been linked to this problem. We consider here neuro-biological, ecological, and evolutionary reasons why bees are particularly vulnerable to these environmental stressors. Central-place foraging on flowers demands advanced capacities of learning, memory, and navigation. However, even at low intensity levels, many stressors damage the bee brain, disrupting key cognitive functions needed for effective foraging, with dramatic consequences for brood development and colony survival. We discuss how understanding the relationships between the actions of stressors on the nervous system, individual cognitive impairments, and colony decline can inform constructive interventions to sustain bee populations.

\section{Bees Are Exposed to Multiple Environmental Stressors}

Bees are ecologically and economically vital pollinators for both wild and cultivated flowers. Presently many populations are in decline [1-4], while demand for pollination crops continues to rise, generating understandable alarm and debate about the 
possibility of an emerging 'pollination crisis' [5]. Many causal factors have been identified, including a range of pathogens and parasites [6,7], human-induced stressors such as pesticides [8-10], and other forms of environmental degradation [11]. Very few of these stressors can be considered new, but many have increased in intensity over the past decade in much of the industrialized world. Our objective in this review is to consider why bees are particularly sensitive to these environmental stressors, even at low levels, and why their populations are now declining.

Bees, with the exception of parasitic species, raise their brood in a single defensible nest [12]. We argue that, in these insects, central-place foraging on ephemeral, dispersed, and highly variable floral resources places particularly heavy demands on cognitive capacities. Individuals must learn to forage at an energetic profit, locate highquality feeding sites, efficiently handle flowers, and navigate back to the nest to provision their brood with the right mix of nectar and pollen. The cognitive capacities underpinning these complex behaviors require optimal devel- opment and function of central brain structures as well as precisely regulated plasticity of brain circuits necessary for learning, memory, and navigation $[13,14]$. These brain systems are very easily disrupted, and it is especially problematic that many pesticides found in floral resources directly target key neural pathways $[15,16]$. Pathogens and nutritional deficits also compromise cognitive functions $[17,18]$. Even mild damage to the brain can significantly reduce foraging performance, thus rendering bees especially vulnerable to these environmental stressors. In social species, such as honey bees, bumblebees, and stingless bees, efficient division of labor and coordination of tasks across nest mates provide buffering against environmental stressors because individuals share a fortress-factory stocked with stored resources [19]. However, this buffering capacity has limits which can be exhausted by frequent stressors. Once this occurs the result is a catastrophic colony decline [20-22].

We develop here a neurobiological, ecological and evolutionary thesis to explain why central- place foraging bees are particularly sensitive to environmental stressors. First we describe the complex cognitive challenges that bees face when foraging, and the neural substrates sup- porting these abilities. Next we review evidence that these essential cognitive abilities are impaired by a range of stressors, ultimately threatening brood development as well as colony function and survival. Finally, we discuss how 
understanding the mechanisms of action of the different stressors and their consequences for individuals and colonies can help to better manage and protect these vital pollinators.

\section{Central-Place Foraging on Flowers Imposes Significant Cognitive Challenges}

Bees must gather large volumes of highly dispersed pollen and nectar, and return with them to the nest to feed their brood [12]. Accordingly, these insects have evolved excellent memory and navigation skills enabling them to exploit complex and variable foraging environments, and more than a century of research has identified the underlying neural circuits $[13,14]$. Although most studies have focused on a few economically important social species, such as honey bees and bumblebees, solitary bees appear to show similar behaviors [12], cognitive capacities [23], and overall brain organization [24]. In the bee brain (Figure 1), visual and olfactory stimuli are first processed by their respective sensory lobes $([25,26]$ for detailed reviews), which then convey information to multisensory integration centers, such as the mushroom bodies (MBs) and the central complex (CX), that are specialized for learning, memory, and spatial navigation tasks, as we describe below.

\section{Learning To Recognize Flowers}

Despite a large variety of available floral species, individual bees tend to forage on the same flower type as long as it provides sufficient nectar or pollen [27]. This floral constancy demonstrates the abilities of bees to learn the association between food rewards and particular floral cues (odor, color, shape, temperature etc.) [28]. In many cases, bees learn more complex associations by generalizing specific floral cues to learn conceptual features common to a range of flowers from the same species [13]. The amount of reward offered by flowers can change very rapidly, and bees can update their learned flower preferences accordingly [29,30]. Bees can also use combinations of floral and social cues, including the presence of conspecifics or other bee species on flowers, to locate and learn rewarding flowers [31].

Many of these mechanisms of learning and memory have been examined in details using experimental approaches (Box 1). For instance, the acquisition of associative 
memories linking floral cues with food rewards relies on changes in neural activity induced by locally coincident activity in the neural networks that process such cues and in those signaling food detection [32]. Plastic changes in connectivity in either the antennal lobes (ALs) or the MBs (Figure 1) can support associative learning about odorants, and both structures modify their activity following learning [26]. In particular, the MBs are required for some complex forms of olfactory learning as well as for the formation of olfactory long-term memory [33,34]. Although less is known about visual learning, there is visual input from optic lobes (OLs) to the MBs (Figure 1), and it is increasingly likely that associative learning of visual features and colors also involves the MBs [35]. Memorizing simple odor-food associations involves excitatory signaling through acetylcholine in the ALs and MBs (Figure 1) [13], a neurotransmitter system specifically targeted by many common pesticides such as neonicotinoids and organophos- phate miticides [15].

\section{Orienting, Navigating, and Learning Places}

Bees use multiple different sources of information to orient [35]. Path integration requires storing of information about distances and directions traveled during the outward journey to be able to plot a direct return path to the nest [36]. Distance is estimated from optic flow [37], which is the movement of the image of the environment across the eye during flight. Direction is determined using the position of the bee relative to the sun [38] and/or the pattern of polarized light in blue sky [39]. Bees possess specialized mechanisms to compensate for the apparent movement of the sun (and the polarization pattern it generates) across the sky during the day [40]. Bees are also sensitive to other global sources of navigational information, such as fine magnetic field variations, and can learn to relate them to local landmarks such that they can still navigate when celestial cues are blocked by cloud [41].

Bees can also learn locations by memorizing visual scenes. They use these stored 'snapshots' for navigation by positional image-matching [36], which compares their current view of the environment with a visual memory of the goal. The degree of matching provides a cue for guidance [42]. Bees form snapshot memories of the nest surroundings on their first foraging attempts outside the nest, and also of the location of food sources [43]. For visual matching, individuals use salient objects (flower patches, trees, buildings), which can be either local cues or panoramic landmarks [36]. 
Honey bees can also perform optic flow matching, using the direction of optic flow caused by major landmarks as a navigational cue [44]. Processing information on optic flow and landmarks while flying demands integrating visual and proprio- ceptive input with a temporal component. Responses to motion stimuli and color are displayed by neurons connecting the OLs to central areas, the lateral protocerebrum (LP) and the MBs [45] (Figure 1), and some of these neurons are involved in visual landmark detection [46].

The functions of the central complex (CX) (Figure 1) are presently poorly understood, but data from other insect species suggest that it is crucial for navigation [35]. In addition to being a likely substrate for a sky compass [38], the CX could also support visual short-term (working) memory and spatial memory [47]. A recent study using a virtual-reality assay (Box 1 ) in Drosophila showed that activity of the ellipsoid body neurons of the CX repre- sented the orientation of the fly relative to visual landmarks [48]. Thus it is increasingly likely that neural activity in the CX contributes to internal representation of position for path integration [48].

\section{Learning Foraging Circuits}

Bees can use their spatial memories dynamically to establish and optimize foraging routes. In nature, foragers must sometimes visit hundreds of patchily distributed flowers to collect sufficient nectar and pollen in a single trip [27], and many species revisit familiar patches over consecutive hours or days in stable sequences called 'traplines' [49]. Recordings of bumblebee flight paths using harmonic radar (Box 1) show that foragers attempt to minimize the overall travel distances between discovered flower patches, a complex optimization task akin to the Traveling Salesman problem [50]. On each new foraging trip, bees try different visitation sequences, ultimately finding (or approximating to) the shortest possible path to visit all patches once, starting and ending at the nest [51]. Route optimization is an iterative improvement process based on learning and memory of flight vectors between feeding locations, supported by path integration and visual guidance [52]. This process allows route flexibility and rapid adjustment of trapline geometry in response to changes in the spatial distribution of floral resources, for instance when a patch becomes depleted or a more rewarding one is discovered [53]. 


\section{Foraging Performance Improves with Foraging Experience}

On their first foraging attempts, bees make orientation flights to systematically acquire information about the nest location without collecting food [54]. Foraging performance then improves over the first week of foraging, likely owing to learned flower identification and handling, and route optimization [55,56]. Dramatic changes in the structure of the adult brain are seen during this period [57]. Foraging activity is reflected by an allometric increase in MB volume [58,59]. In honey bees this expansion is caused by increased dendritic arborization of $\mathrm{MB}$ intrinsic neurons receiving visual and olfactory input accompanied by pruning of micro- glomeruli (synaptic boutons) $[57,60]$, partly due to the activation of cholinergic receptors [61]. The selective localization of these structural changes suggests activity-dependent synaptic plasticity as an underlying mechanism [57]. Dendritic growth can provide a substrate for the formation of new synapses to support stable memories [62]. At the same time selective growth and pruning of connections is thought to optimize the performance of brain centers in the rich visual and olfactory environments experienced during foraging [57].

\section{Stressors Affect Brain Functions, Cognition, and Behavior}

Successful foraging is based on the precise integration of information processed across the major brain networks, as well as dynamic structural modifications of such networks. Therefore even subtle disturbances of neural function could have dramatic consequences on individual cognitive abilities and hence foraging performance. From this perspective it is a major concern that most of the stressors presently impacting on bees target the brain. The range of stressors has been well reviewed previously $[1,11]$. We emphasize here how many of these impair cognitive abilities and foraging performance at exposure levels far below those that kill the bee.

\section{Pesticides and Heavy Metals}

Many pesticides affect bee cognition. In recent years, neonicotinoid insecticides have drawn the most attention [63]. These insecticides disrupt cholinergic transmission, the main excitatory pathway in the insect brain, vital for effective learning and synaptic plasticity $[13,26]$. While acute exposure to very small doses of neonicotinoids has been shown to inactivate MB neurons [15], chronic exposure can impair development of the 
entire MB $[16,64]$. These effects almost certainly explain the dramatic impacts of sublethal doses of neonicotinoids on learning and memory in honey bees [65], bumblebees [66], and solitary bees [23], which can be linked to deficits in MB plasticity [16]. Pesticide exposure also disrupts visuospatial memory and navigation $[9,67,68]$, most likely through disruption of processing in the corresponding path- ways (Figure 1 ), but this has yet to be demonstrated. Alarmingly, bees learn to prefer nectar containing neonicotinoids over non-contaminated nectar because of incidental actions of pesticides on the nicotinic receptors involved in reward processing [69].

Fipronil, a widely used insecticide and acaricide, targets neuronal receptors involved in inhibitory transmission by g-aminobutyric acid (GABA) and glutamate [70]. In honey bees GABA signaling is vital for normal MB function, particularly for complex learning [33,71]. Acute fipronil treatment severely reduces olfactory learning and memory performance [72]. Additional indications of neuronal cell death in the MBs following fipronil exposure suggest possible long- term cognitive impairments in honey bees [73] and stingless bees [74].

Some pesticides contain manganese, which induces precocious foraging in honey bees [75]. Its effect on sucrose responsiveness suggests that it interferes with signaling pathways important for associative learning, as indicated by the abundant expression of a manganese transporter in MBs and ALs [75] (Figure 1). Selenium, another heavy metal found in crop treatments, has been found to change sucrose responsiveness, olfactory learning, and long- term memory [76].

\section{Parasites and Pathogens}

Human activities have intensified the pressures of parasites and pathogens on bees through dispersion of bacteria, viruses, fungi, and mites across the world [11]. While few parasites or pathogens act directly on the brain, many have a strong impact on the behavior of bees [6]. Part of this can be explained by the activation of the immune system, which might interfere with energy supply or signaling mechanisms. Even an immune response induced by non-patho- genic molecules can reduce olfactory associative learning abilities $[77,78]$. 
The microsporodian Nosema cerana and the mite Varroa destructor are two major parasites of honey bees. Exposure to either parasite induces specific but overlapping patterns of altered gene expression in the brain of their host [79]. Varroa infection alters brain expression of many genes involved in neurotransmitter signaling, including through GABA [79]. These impacts on the brain are thought to induce poor navigation performance by infected bees $[80,81]$.

Varroa carries many viruses, and a Varroa infection of a colony is a complex syndrome of many co-associated pathogens. Some the effects of varroensis are due to viral infections $[7,79]$. For example, the deformed wing virus (DWV) impacts on olfactory learning, possibly by targeting brain areas of importance for foraging [18]. Although there is no known impact of DWV on bee visual learning and navigation, other viruses, such as the Israeli acute paralysis virus (IAPV), affect homing behavior [82].

\section{Malnutrition}

Intensive farming and the expansion of monocultures have imposed strong constraints on the dietary diversity of bees because significantly fewer food resources are available to them, often during limited flowering seasons [11]. Bee nutrition is partitioned between nectar, the main source of carbohydrates, and pollen, which provides proteins, lipids, vitamins, and other micronutrients [83]. Limited food intake reduces performance in a simple learning task [78], but having enough food is not necessarily sufficient for optimal cognitive processing. In honey bees, olfactory associative learning is disrupted by qualitative changes in essential lipids [17] or amino acids [84]. Pollen shortage during development can also lead adults to forage earlier and for a shorter period [85], whereas nectar deprivation increases impulsive, suboptimal food choices [86].

\section{From Reduced Foraging Performance to Colony Collapse}

Few of the stressors we have considered would kill bees outright at ecological levels. None- theless, impairment of the cognitive abilities and food collection performance by low stresses can have extremely severe consequences on bee functions and survival, and crucially on their capacity to successfully rear brood and maintain colonies. Hence these stresses can have very significant impacts on populations. 
Comparative research on bee declines suggests that the resilience of bees to stressors depends on their level of sociality $[2,87]$, although this needs to be confirmed by further studies (see Outstanding Questions). In principle, solitary bees are the most vulnerable because reduced foraging efficiency of the female following stress exposure immediately jeopardizes the development of her brood. These species lack the profusion of specialized group behaviors observed in social bees (e.g., corpses and diseased brood removal, social fever, collection of antimicrobial and antiviral plant resins) that can mitigate the impact of pathogen stressors on colonies [88].

However, the stress tolerance of social bees is not without limits stressors, even at low levels, can also have extremely severe consequences on colonies. In the most social species, such as honey bees, foraging is undertaken by middle-aged adults that have completed a period of orientation flights and brain maturation to prepare them for the cognitive demands of foraging [58,59]. Stressors not only disrupt foraging performance, but also the process of preparing for foraging. For honey bees, a very common response to many stressors is to begin foraging prematurely [21] (Figure 2). It has been argued that delaying high-risk tasks to later in life is an effective strategy to extend mean longevity of workers and increase their total contribution to the colony [89]. Nevertheless, if worker lifespan is reduced, workers react by proportionally compressing their time allocation to each task, and commence foraging early. This is likely to be an adaptive response to acute stress because it would temporarily compensate for the diminished foraging effort of the colony. However, in conditions of prolonged stress, this response can accelerate colony decline because bees that start foraging precociously complete fewer trips in their lifetime [90] and live less long [21].

Simulation models suggest that continuous stress can create a situation in which the foraging force is dominated by precocious foragers [21,91], and then becomes so inefficient that it can no longer support the colony, at which point the colony population dramatically collapses (Figure 2). Stressed bumblebee colonies, although smaller and socially simpler than honey bee colonies, also show highly non-linear responses to environmental stressors $[10,20]$. Various impairments of colony function (including foraging, but also thermoregulation, defense, and hygienic behavior) can generate changes in population dynamics via feedback loops affecting rates of hatching and adult death, sometimes leading to colony collapse [20]. These complex dynamics 
might explain the observed widespread declines of wild and managed bee populations [1-4]. The known stressors of bees are not new, and many populations have been in a steady decline for decades, but the accelerated declines described recently suggest that we are now reaching the point at which the cumulative stress on colonies is exceeding their tolerance capacity [11].

How then can the agricultural environ- ment be managed to ensure bees receive adequate nutrition from diverse floral sources? Can we design nutri- tionally optimized plant assemblages to preserve bee populations? Crops provide huge amounts of foods, but these plants that have been selected to optimize production [295_TD\$DIFF][278_TD\$DIFF]typically yield poor quality diets to bees [99]. Research is necessary to quantify the precise nutrient needs of bees, how they vary across colony developmental stages, species, and in the face of specific stressors, and their impact on behavior and cognition.

\section{Concluding Remarks and Future Prospects}

Central-place foraging bees are particularly vulnerable to many current environmental stressors. These insects have evolved refined cognitive abilities enabling them to effectively exploit complex and changing foraging environments to provision their nest. Such capacities demand the optimal function and coordination of major systems in the small bee brain. Many stressors disrupt brain function, with the consequence of reduced foraging performance, ultimately compromising the brood or whole colonies. These gradual and pervasive effects might explain why eco-toxicological studies, alone, have failed to provide accurate predictions of how stressors can damage bee colonies. We therefore argue that more integrated research that considers actions of the different stressors on bee behavior, cognition, and colony function is urgently needed to understand the declines of these major pollinators and manage their populations see Outstanding Questions).

Pesticides provide an informative case in point. Agriculture has become increasingly reliant on 'next-generation' neonicotinoid pesticides because they are so effective at killing pest insects at low doses by directly targeting the insect central nervous system [8]. Recent research describing the neural impacts, behavioral impairments, and changes in colony dynamics at field contamination levels by pesticides $[8-10,55,68]$ 
has forced a re-evaluation of the 'safe-level' of pesticide exposure for individual bees and colonies [63]. Using this new knowledge we must now determine how pesticides can be managed in the agricultural landscape in a manner that is compatible with sustaining bee populations. Many other stressors contribute to colony decline [1,11], for which the precise mechanisms of action need to be unraveled.

As discussed above, the stress tolerance of a colony is not without limits and, given the increase in bee declines seen in the past decade, it would appear we are very close to exhausting those limits for some key pollinating bee species. Even so, this is far from a hopeless story. Combining conceptual and methodological advances in neuroscience, ecology, and evolutionary biology can bring significant insights into how specific stressors affect bee behavior and colony dynamics, and help to identify ecological interventions to ameliorate stress on bees. Most of the stressors damaging bee populations are human-induced, and can be reduced or eliminated from the environment if there is sufficient will and/or economic imperative.

\section{Acknowledgments}

This work was funded by the CNRS. The research was also supported by PhD fellowships of the French Ministry of Research to S.K. and A.C., an Agence Nationale de la Recherche (ANR) grant (ANR-13-ADAP-0002) to J.M.D., an Australian Research Council (ARC) Future Fellowship to A.B.B., and ANR (ANR-16-CE02-0002-01), IDEX, and Fyssen grants to M.L.

\section{References}

1. Potts, S.G. et al. (2010) Global pollinator declines: trends, impacts and drivers. Trends Ecol. Evol. 25, 345-353

2. Rundlöf, M. et al. (2015) Seed coating with a neonicotinoid insecticide negatively affects wild bees. Nature $521,77-80$

3. Ollerton, J. et al. (2014) Extinctions of aculeate pollinators in Britain and the role of large-scale agricultural changes. Science 346, 1360-1362

4. Woodcock, B.A. et al. (2016) Impacts of neonicotinoid use on longterm population changes in wild bees in England. Nat. Commun. 7, 12459 
5. Holden, C. (2006) Report warns of looming pollination crisis in North America. Science 314, 397

6. Cornman, R.S. et al. (2012) Pathogen webs in collapsing honey bee colonies. PLoS One 7, e43562

7. Francis, R.M. et al. (2013) Varroa-virus interaction in collapsing honey bee colonies. PLoS One 8, e57540

8. Van der Sluijs, J.P. et al. (2013) Neonicotinoids, bee disorders and the sustainability of pollinator services. Curr. Opin. Environ. Sustain. 5, 293-305

9. Henry, M. et al. (2012) A common pesticide decreases foraging success and survival in honey bees. Science 336, 348-350

10. Gill, R. et al. (2012) Combined pesticide exposure severely affects individualand colony-level traits in bees. Nature 491, 105-108

11. Goulson, D. et al. (2015) Bee declines driven by combined stress from parasites, pesticides, and lack of flowers. Science 347, 1255957

12. Michener, C.D. (2000) The Bees of the World, The John Hopkins University Press

13. Giurfa, M. (2013) Cognition with few neurons: higher-order learning in insects. Trends Neurosci. 36, 285-294

14. Menzel, R. (2012) The honeybee as a model for under- standing the basis of cognition. Nat. Rev. Neurosci. 13, 758-768

15.Palmer, M.J. et al. (2013) Cholinergic pesticides cause mush- room body neuronal inactivation in honeybees. Nat. Commun. 4, 1634-1642

16.Peng, Y.-C. and Yang, E.-C. (2016) Sublethal dosage of imida- 44. cloprid reduces the microglomerular density of honey bee mushroom bodies. Sci. Rep. 6, 19298-19311

17. Arien, Y. et al. (2015) Omega-3 deficiency impairs honey bee learning. Proc. Natl. Acad. Sci. USA 112, 201517375

18. Iqbal, J. and Mueller, U. (2007) Virus infection causes specific learning deficits in honeybee foragers. Proc. R. Soc. B. 274, 1517-1521

19. Hölldobler, B. and Wilson, E.O. (2009) The Superorganism: The Beauty, Elegance, and Strangeness of Insect Societies, WW Norton \& Company

20. Bryden, J. et al. (2013) Chronic sublethal stress causes bee colony failure. Ecol. Lett. 16, 1463-1469 
21.Perry, C.J. et al. (2015) Rapid behavioral maturation accelerates 49. failure of stressed honey bee colonies. Proc. Natl. Acad. Sci. USA 112, 3427-3432

22. Khoury, D.S. et al. (2011) A quantitative model of honey bee colony population dynamics. PLoS One 6, e18491

23. Jin, N. et al. (2015) The neonicotinoid clothianidin interferes with navigation of the solitary bee Osmia cornuta in a laboratory test. J. Exp. Biol. 218, 2821-2825

24.Farris, S.M. (2016) Insect societies and the social brain. Curr. Opin. Insect Sci. $15,1-8$

25.Dyer, A.G. et al. (2011) Colour processing in complex environtors of tropical plants. Science 171, 203-205

26.Sandoz, J.C. (2011) Behavioral and neurophysiological study of olfactory perception and learning in honeybees. Front. Syst. Neurosci. 5, 98-108 54.

27.von Frisch, K. (1966) The Dancing Bees: An Account of the Life and Senses of the Honey Bee, Methuen 55.

28. Chittka, L. et al. (1999) Flower constancy, insect psychology, and plant evolution. Naturwissenschaften $86,361-377$

29.Dyer, a.G. et al. (2014) Bee reverse-learning behavior and intra- 56. colony differences: Simulations based on behavioral experi- ments reveal benefits of diversity. Ecol. Modell. 277, 119-131 57.

30. Raine, N.E. and Chittka, L. (2012) No trade-off between learning speed and associative flexibility in bumblebees: a reversal learning test with multiple colonies. PLoS One 7, e45096 58.

31.Dawson, E.H. et al. (2013) Learning by observation emerges from simple associations in an insect model. Curr. Biol. 23, 59.

32. Giurfa, M. and Sandoz, J.-C. (2012) Invertebrate learning and memory: fifty years of olfactory conditioning of the proboscis 60 . extension response in honeybees. Learn. Mem. 19, 54-66

33. Devaud, J. et al. (2015) Neural substrate for higher-order learning in an insect: Mushroom bodies are necessary for configural discriminations. Proc. Natl. Acad. Sci. USA 112, E5854-E5862 61.

34. Menzel, R. (1999) Memory dynamics in the honeybee. J. Comp. Physiol. A 185, $323-340$ 
35. Webb, B. and Wystrach, A. (2016) Neural mechanisms of insect 62. navigation. Curr. Opin. Insect Sci. 15, 27-39

36. Collett, M. et al. (2013) Spatial memory in insect navigation. Curr. Biol. 23, R789-R800

37. Srinivasan, M.V. (2000) Honeybee navigation: nature and cali- bration of the 'odometer'. Science 287, 851-853

38. el Jundi, B. et al. (2014) Integration of polarization and chromatic cues in the insect sky compass. J. Comp. Physiol. A 200, 575-589

39. Dovey, K.M. et al. (2013) The depth of the honeybee's backup sun-compass systems. J. Exp. Biol. 216, 2129-2139

40.Zeller, M. et al. (2015) Transmedulla neurons in the sky compass network of the honeybee (Apis mellifera) are a possible site of circadian input. PLoS One 10, e0143244

41. Wajnberg, E. et al. (2010) Magnetoreception in eusocial insects: an update. J. R. Soc. Interface 7, S207-S225

42. Collett, T.S. and Collett, M. (2002) Memory use in insect visual navigation. Nat. Rev. Neurosci. 3, 542-552

43. Philippides, A. et al. (2013) Bumblebee calligraphy: the design and control of flight motifs in the learning and return flights of Bombus terrestris. J. Exp. Biol. 216, 1093-1104

44. Dittmar, L. et al. (2010) Goal seeking in honeybees: matching of optic flow snapshots? J. Exp. Biol. 213, 2913-2923

45.Paulk, A.C. et al. (2009) Visual processing in the central bee brain. J. Neurosci. 29, 9987-9999

46. Mertes, M. et al. (2014) Visual motion-sensitive neurons in the bumblebee brain convey information about landmarks during a navigational task. Front. Behav. Neurosci. 8, 1-13

47.Pfeiffer, K. and Homberg, U. (2014) Organization and functional roles of the central complex in the insect brain. Annu. Rev. Entomol. 59, 165-184

48. Seelig, J.D. and Jayaraman, V. (2015) Neural dynamics for landmark orientation and angular path integration. Nature 521, 186-191

49. Janzen, D.H. (1971) Euglossine bees as long-distance pollinators. Science. 171, 203-205 
50.Lihoreau, M. et al. (2013) Unravelling the mechanisms of trapline foraging in bees. Commun. Integr. Biol. 6, e22701

51.Lihoreau, M. et al. (2010) Travel optimization by foraging bumblebees through readjustments of traplines after discovery of new feeding locations. Am. Nat. 176, 744-757

52. Reynolds, A.M. et al. (2013) A simple iterative model accurately captures complex trapline formation by bumblebees across spatial scales and flower arrangements. PLoS Comput. Biol. 9, e1002938

53. Lihoreau, M. et al. (2012) Radar tracking and motion-sensitive cameras on flowers reveal the development of pollinator multi- destination routes over large spatial scales. PLoS Biol. 10, e1001392

54.Degen, J. et al. (2015) Exploratory behaviour of honeybees during orientation flights. Anim. Behav. 102, 45-57

55. Gill, R.J. and Raine, N.E. (2014) Chronic impairment of bumble- bee natural foraging behaviour induced by sublethal pesticide exposure. Funct. Ecol. 28, 1459-1471

56. Dukas, R. (2008) Life history of learning: performance curves of honeybees in the wild. Ethology 114, 1195-1200

57. Muenz, T.S. et al. (2015) Neuronal plasticity in the mushroom body calyx during adult maturation in the honeybee and possible pheromonal influences. Dev. Neurobiol. 75, 1368-1384

58. Withers, G.S. et al. (1993) Selective neuroanatomical plasticity and division of labour in the honeybee. Nature 364, 238-240

59. Jones, B.M. et al. (2013) Plasticity of the worker bumblebee brain in relation to age and rearing environment. Brain. Behav. Evol. 82, 250-261

60. Groh, C. et al. (2012) Age-related plasticity in the synaptic ultrastructure of neurons in the mushroom body calyx of the adult honeybee Apis mellifera. J. Comp. Neurol. 520, 3509- 3527

61. Ismail, N. et al. (2006) Stimulation of muscarinic receptors mimics experiencedependent plasticity in the honey bee brain. Proc. Natl. Acad. Sci. USA 103, 207-211

62. Hourcade, B. et al. (2010) Long-term memory leads to synaptic reorganization in the mushroom bodies: a memory trace in the insect brain? J. Neurosci. 30 , $6461-6465$ 
63. Godfray, H.C.J. et al. (2015) A restatement of recent advances in the natural science evidence base concerning neonicotinoid insecticides and insect pollinators. Proc. R. Soc. B 282, 20151821

64.van Tomé, H.V. et al. (2012) Imidacloprid-induced impairment of mushroom bodies and behavior of the native stingless bee Melipona quadrifasciata anthidioides. PLoS One 7, e38406

65. Tan, K. et al. (2015) A neonicotinoid impairs olfactory learning in Asian honey bees (Apis cerana) exposed as larvae or as adults. Sci. Rep. 5, 10989

66. Stanley, D.A. et al. (2015) Bumblebee learning and memory is impaired by chronic exposure to a neonicotinoid pesticide. Sci. Rep. 5, 16508

67. Fischer, J. et al. (2014) Neonicotinoids interfere with specific components of navigation in honeybees. PLoS One 9, e91364

68. Stanley, D.A. et al. (2016) Investigating the impacts of field- realistic exposure to a neonicotinoid pesticide on bumblebee foraging, homing ability and colony growth. J. Appl. Ecol. 53, 1440-1449

69. Kessler, S.C. et al. (2015) Bees prefer foods containing neon- icotinoid pesticides. Nature $521,74-76$

70. Simon-Delso, N. et al. (2015) Systemic insecticides (neonicoti- noids and fipronil): trends, uses, mode of action and metabo- lites. Environ. Sci. Pollut. Res. Int. 22, 5-34

71. Boitard, C. et al. (2015) GABAergic feedback signaling into the calyces of the mushroom bodies enables olfactory reversal learning in honey bees. Front. Behav. Neurosci. 9, 1-13

72. Bernadou, A. et al. (2009) Effect of fipronil on side-specific antennal tactile learning in the honeybee. J. Insect Physiol. 55, 1099-1106

73. Roat, T.C. et al. (2013) Effects of sublethal dose of fipronil on neuron metabolic activity of africanized honeybees. Arch. Envi- ron. Contam. Toxicol. 64, 456466

74.Jacob, C.R. et al. (2015) Impact of fipronil on the mushroom bodies of the stingless bee Scaptotrigona postica. Pest Manag. Sci. 71, 114-122

75. Søvik, E. et al. (2015) Negative impact of manganese on hon- eybee foraging. Biol. Lett. 11, 20140989 
76.Burden, C.M. et al. (2016) Acute exposure to selenium disrupts associative conditioning and long-term memory recall in honey bees (Apis mellifera). Ecotoxicol. Environ. Saf. 127, 71-79

77. Alghamdi, a. et al. (2008) Immune response impairs learning in free-flying bumble-bees. Biol. Lett. 4, 479-481

78. Jaumann, S. et al. (2013) Energetic cost of learning and memory can cause cognitive impairment in honeybees. Biol. Lett. 9, 20130149

79. McDonnell, C.M. et al. (2013) Ecto- and endoparasite induce similar chemical and brain neurogenomic responses in the honey bee (Apis mellifera). BMC Ecol. 13, 25

80. Wolf, S. et al. (2016) Optimal search patterns in honeybee orientation flights are robust against emerging infectious dis- eases. Sci. Rep. 6, 32612

81. Wolf, S. et al. (2014) So near and yet so far: harmonic radar reveals reduced homing ability of Nosema infected honeybees. PLoS One 9, e103989

82. Li, Z. et al. (2013) Viral infection affects sucrose responsiveness and homing ability of forager honey bees, Apis mellifera L. PLoS One 8, e77354

83. Vaudo, A.D. et al. (2016) Macronutrient ratios in pollen shape bumble bee (Bombus impatiens) foraging strategies and floral preferences. Proc. Natl. Acad. Sci. USA 113, E4035-E4042

84.Simcock, N.K. et al. (2014) Single amino acids in sucrose rewards modulate feeding and associative learning in the hon- eybee. J. Insect Physiol. 69, 41-48

85.Scofield, H.N. and Mattila, H.R. (2015) Honey bee workers that are pollen stressed as larvae become poor foragers and waggle dancers as adults. PLoS One 10, e0121731

86. Mayack, C. and Naug, D. (2015) Starving honeybees lose self- control. Biol. Lett. 11, 20140820

87.Cresswell, J.E. et al. (2012) Dietary traces of neonicotinoid pesticides as a cause of population declines in honey bees: an evaluation by Hill's epidemiological criteria. Pest Manag. Sci. 68, 819-827

88. Cremer, S. et al. (2007) Social Immunity. Curr. Biol. 17, 693-702 89.

89. Woyciechowski, M. and Moron , D. (2009) Life expectancy and onset of foraging in the honeybee (Apis mellifera). Insectes Soc. 56, 193-201 
90. Ushitani, T. et al. (2015) Accelerated behavioural development changes finescale search behaviour and spatial memory in honey bees (Apis mellifera $L$ ). J. Exp. Biol. 219, 412-418

91.Becher, M.A. et al. (2014) BEEHAVE: a systems model of honeybee colony dynamics and foraging to explore multifactorial causes of colony failure. J. Appl. Ecol. 51, 470-482

92. Junca, P. and Sandoz, J.C. (2015) Heat perception and aversive learning in honey bees: putative involvement of the thermal/ chemical sensor AmHsTRPA. Front. Physiol. 6, 316

93.Lichtenstein, L. et al. (2015) Dumb and lazy? A comparison of color learning and memory retrieval in drones and workers of the buff-tailed bumblebee, Bombus terrestris, by means of per conditioning. PLoS One 10, e0134248

94. Srinivasan, M.V. (2014) Going with the flow: a brief history of the study of the honeybee's navigational 'odometer'. J. Comp. Physiol. A 200, 563-573

95. Portelli, G. et al. (2011) Honeybees' speed depends on dorsal as well as lateral, ventral and frontal optic flows. PLoS One 6, e19486

96. Crall, J.D. et al. (2015) BEEtag: A low-cost, image-based track- ing system for the study of animal behavior and locomotion. PLoS One 10, e0136487

97.Paulk, A.C. et al. (2014) Selective attention in the honeybee optic lobes precedes behavioral choices. Proc. Natl. Acad. Sci. USA 111, 5006-5011

98. Taylor, G.J. et al. (2013) Vision and air flow combine to stream- line flying honeybees. Sci. Rep. 3, 2614

99.Vaudo, A.D. et al. (2015) Bee nutrition and floral resource res- toration. Curr. Opin. Insect Sci. 10, 133-141

100. Tenczar, P. et al. (2014) Automated monitoring reveals extreme interindividual variation and plasticity in honeybee foraging activ- ity levels. Anim. Behav. 95, 41-48 


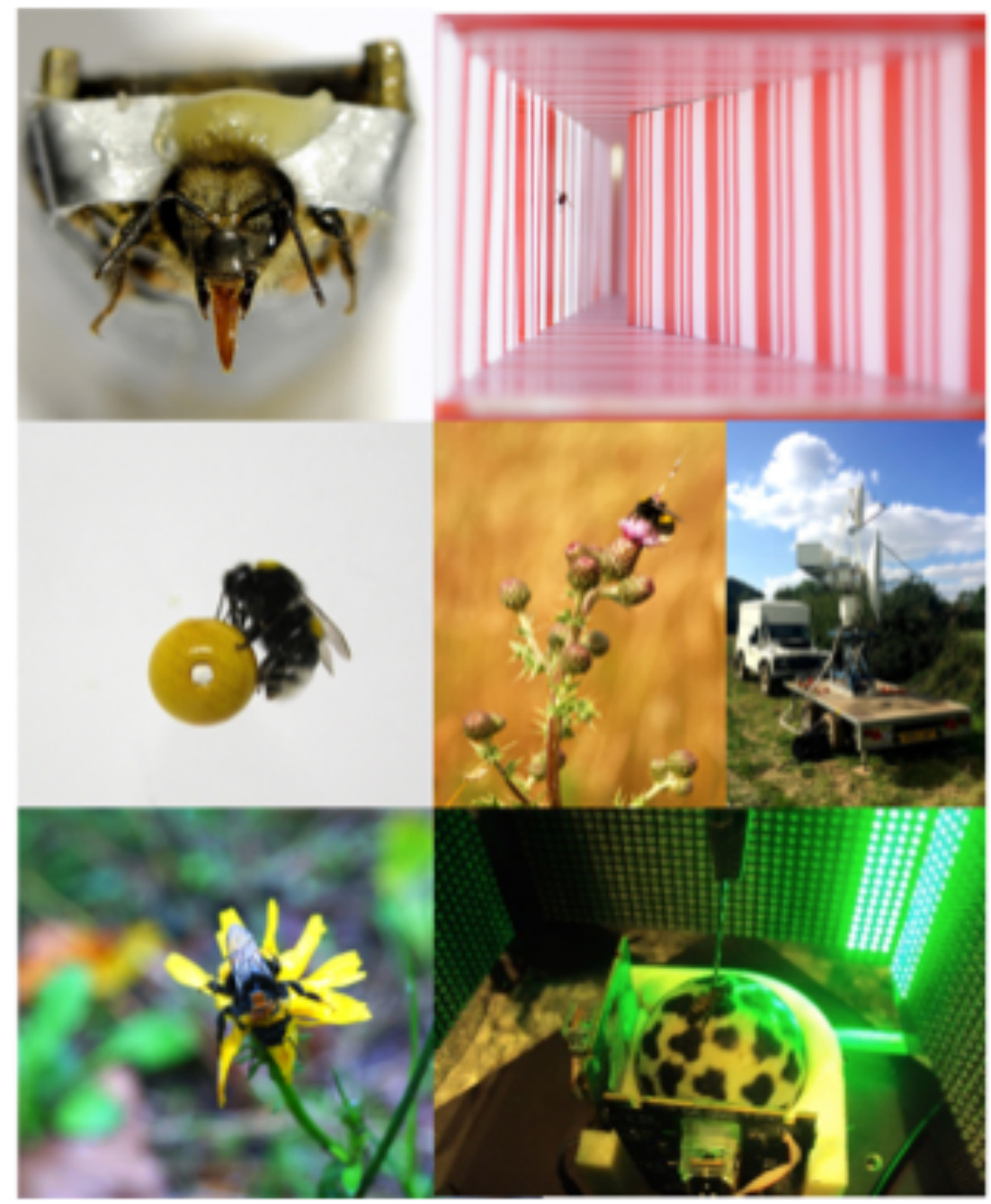

Figure I. Methods for Studying Bee Learning and Memory. (A) Restrained honey bee showing proboscis extension reflex (PER) (C. Fresillon/CNRS). (B) Free-flying honey bee in a flight tunnel covered with visual patterns generating optic flow ( $F$. Vrignaud/DGA) [95]. (C) Bumblebee foraging on an artificial flower (M. Lihoreau). (D) (Left) Bumblebee with a radar transponder in the field (J.L. Woodgate); (Right) harmonic radar (J.C. Makinson). (E) Bumblebee with an RFID tag in the field (S. Klein). (F) Tethered honey bee walking on a locomotion compensator in a controlled visual environment displayed onto LED panels (G.J. Taylor) [97]. 


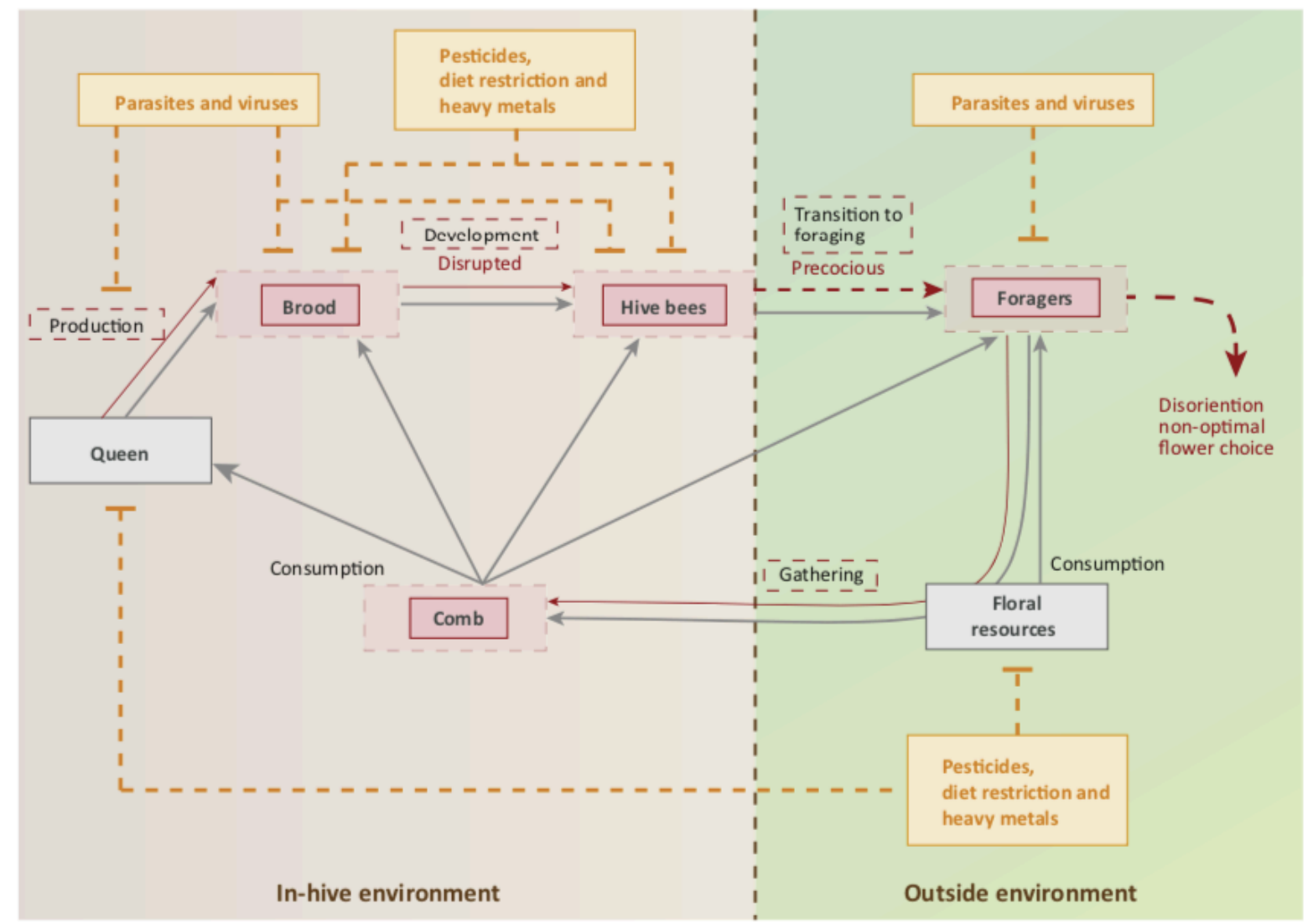

Figure 2. Effects of Stressors on Honey Bee Colony Dynamics. In a non-stressed colony (grey arrows), the brood (eggs, larvae, and pupae) develops into in-hive bees (e.g., nurses) that begin to forage 2 weeks later. Foragers gather nectar and pollen from floral resources for storage in the hive (comb). The food stock is consumed by the queen, the larvae, the in-hive bees, and the foragers. Individual bees can be exposed to environmental stressors (orange boxes) at different stages, potentially disrupting the dynamics of the whole colony. Stressors reduce brood production, alter development, induce precocious foraging onset of in-hive bees, and affect the cognitive performance of foragers, leading to disorientation and less-efficient food gathering (red arrows). The synergistic action of stressors at different levels of this complex system can lead to dramatic colony collapse. Plain red arrows indicate quantitative changes. Broken red arrows indicate qualitative changes. Adapted from [22].

Box 1. Studying the Mechanisms of Learning and Memory in Bees 
Experimental work addressing the fine-scale neural and behavioral bases of cognitive capacities in bees has relied primarily on Pavlovian conditioning in which an individual is trained to associate an initially neutral stimulus (the conditioned stimulus, CS) with an unconditioned stimulus (US) that elicits an innate response [13,14]. Learning the CS-US association leads the animal to respond to the CS. Historically, the dominant paradigm has been the appetitive conditioning (using a sugar solution as the US) of the proboscis (tongue) extension reflex (PER) using a restrained bee (Figure IA) [32], although aversive paradigms also exist [92]. This method allows study of elemental associations between two prescribed events, and also non-elemental associations (when individuals respond in an adaptive manner to novel stimuli using learned information in a new context). In recent years considerable progress has been made by combining PER conditioning with pharmacological treatments, electrophysiological recordings, and brain functional imaging to unravel mechanisms of learning and memory, especially for olfactory learning [33].

So far, attempts at associative conditioning of visual CS in PER conditioning with restrained bees have yielded low performance levels [93]. By contrast, impressive visual learning capacities have been described using free-flight assays in which bees obtain a sugar reward if they make a correct choice when learning to navigate in a maze (Figure IB) [94,95] or foraging in arrays of artificial flowers (Figure IC) $[30,31,51,77]$. Automated tracking systems such as harmonic radars (Figure ID) $[53,67,80,81]$, radio-frequency identification (RFID) (Figure IE) $[9,10,21,55,68]$, and computer vision [96] allow precise quantification of behavioral data in laboratory or semi-field conditions. These approaches have revealed the cognitive abilities of bees in learning complex visual features and relational properties between stimuli [13]. New developments in virtual-reality assays, in which tethered bees walk on a locomotion compensator (Figure IF) [97] or fly [98] to make foraging decisions in response to stimuli displayed on a screen, hold considerable promise to explore the neural mechanisms of visual learning and navigation.

\section{Outstanding Questions}


What are the points of greatest vulnerability in the bee brain? Neurogenomic profiling has started to provide an over- view of the gene expression changes occurring in the brain in response to pathogens [79], but we have yet to understand the signaling pathways involved and the functional relevance of these changes. More integrative work is now necessary to identify pre- cisely how stressors damage the brain to reduce foraging performance. This must couple genomic studies with functional analyses of changes in circuit performance and behavior. Understanding these fundamental processes in the developing and adult brain will help designing neuroprotective treatments to improve the resilience of managed bees.

Are all bee species similarly vulnerable to stressors? Bees greatly vary in their social organization (from solitary to social), feeding ecology, and habitats [12]. While most attention has focused on managed populations of generalist species with a social lifestyle, such as honey bees and bumblebees, comparative research is now needed to assess the general impact of stressors on the wide diversity of pollinators.

How can pesticides and bees be man- aged to keep populations at a 'safe level' of exposure? A key issue is determining what cocktails and levels of pesticide exposure populations can tolerate. Often there are multiple different pesticides at use in the landscape. We need more information on how these chemicals might accumulate and persist in nests, and how they interact to impact on bee physiology and behavior.

How then can the agricultural environment be managed to ensure bees receive adequate nutrition from diverse floral sources? Can we design nutritionally optimized plant assemblages to preserve bee populations? Crops provide huge amounts of foods, but these plants that have been selected to optimize production typically yield poor quality diets to bees [99]. Research is necessary to quantify the precise nutrient needs of bees, how they vary across colony developmental stages, species, and in the face of specific stressors, and their impact on behavior and cognition.

Can the pollination performance of managed social bees (honey bees and bumblebees) be sustainably improved by manipulating colony composition? Within a colony, social bees show high levels of interindividual behavioral and cognitive variability. In honey bees a small number of individuals complete a disproportionately high number of foraging trips [100]. Characterizing this variability between bees, what 
causes it, and how it changes under stress conditions will be necessary to understand the con- sequences of environmental stressors on the resilience of colonies. 